\title{
Modeling of Coaxial Slot Waveguides Using Analytical and Numerical Approaches: Revisited
}

\author{
Kok Yeow You, ${ }^{1}$ Jamaliah Salleh,, ${ }^{1}$ Mohd Fareq Abd Malek, ${ }^{2}$ Zulkifly Abbas, ${ }^{3}$ \\ Cheng Ee Meng, ${ }^{4}$ and Lee Kim Yee ${ }^{5}$ \\ ${ }^{1}$ Department of Radio Communication Engineering, Faculty of Electrical Engineering, Universiti Teknologi Malaysia, \\ 81310 Skudai, Malaysia \\ ${ }^{2}$ School of Electrical Systems Engineering, Universiti Malaysia Perlis, 02600 Perlis, Malaysia \\ ${ }^{3}$ Department of Physics, Faculty of Science, Universiti Putra Malaysia, 43400 Serdang, Malaysia \\ ${ }^{4}$ School of Mechatronic, Universiti Malaysia Perlis, 02600 Perlis, Malaysia \\ ${ }^{5}$ Department of Electrical and Electronic Engineering, Faculty of Engineering and Science, Universiti Tunku Abdul Rahman, \\ 46200 Selangor, Malaysia
}

Correspondence should be addressed to Kok Yeow You, kyyou@fke.utm.my

Received 10 August 2011; Revised 29 November 2011; Accepted 1 January 2012

Academic Editor: Andres Peratta

Copyright () 2012 Kok Yeow You et al. This is an open access article distributed under the Creative Commons Attribution License, which permits unrestricted use, distribution, and reproduction in any medium, provided the original work is properly cited.

\begin{abstract}
Our reviews of analytical methods and numerical methods for coaxial slot waveguides are presented. The theories, background, and physical principles related to frequency-domain electromagnetic equations for coaxial waveguides are reassessed. Comparisons of the accuracies of various types of admittance and impedance equations and numerical simulations are made, and the fringing field at the aperture sensor, which is represented by the lumped capacitance circuit, is evaluated. The accuracy and limitations of the analytical equations are explained in detail. The reasons for the replacement of analytical methods by numerical methods are outlined.
\end{abstract}

\section{Introduction}

Since the 1940s, coaxial slot waveguides have been used as antennas and as cables for electric power. Recently, this type of waveguide has been used in dielectric measurements and the treatment of cancer. Hence, a theoretical formula was needed to design and model the waveguide. Many theoretical formulas have been developed and derived in the past 70 years. Indeed, in the past, the analytical formulas were an important tool for waveguide design because few numerical methods were available and advance computer equipment and software were not yet available to reduce the costs associated with experimental design. Until the 1960s, many reliable numerical techniques were developed and applied for solving electromagnetic problems, such as the finite difference time-domain (FDTD) method [1], the moment method (MoM) [2], and the finite element method (FEM) [3]. At that time, 2D numerical techniques were used to analyze coaxial waveguides because of their simplicity and the symmetrical shapes they produced.

In reality, no completely analytical solutions exist due to the difficulty of deriving the formulas and the many unknown environmental factors that are not included in the formulation. In the author's view, analytical formulas referred to equations that were based on theoretical concepts and that had no unknown variables. Although the complicated analytical formulas were solved with the aid of numerical methods, such as series expansion and the trapezium rule, the solutions were still obtained by an analytical approach. When a theoretical formula has a matrix of unknown variables and is solved by using a modern numerical routine, such as FEM and MoM, we will define it as a numerical approach. In fact, modern numerical methods can produce results that are close to practical measurements because they divide the problem into many small segments and solve them one by one. 


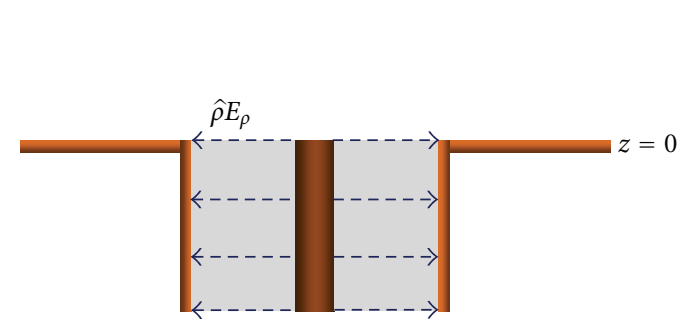

(a)

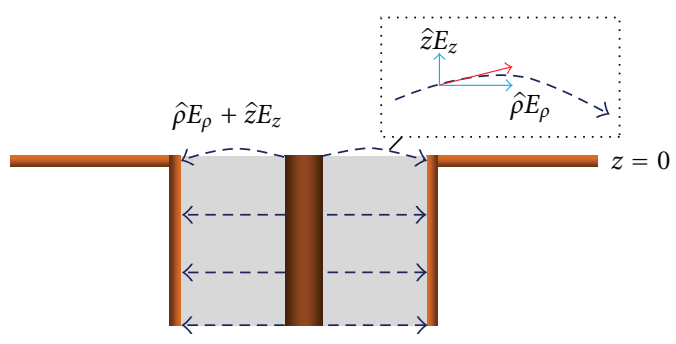

(b)

Figure 1: Propagated wave from coaxial line $(z<0)$ into free space $(z>0)$ through the ground plane $(z=0)$.

However, the development of powerful numerical techniques does not mean that analytical formulas are not still useful in predicting waveguide performance, and, in addition, many of numerical models are based on analytical formulations. For instance, many online broad-band dielectric measurements involve the prediction of the dielectric properties of samples based on measurements of electric signals, but, unfortunately, those numerical techniques are not suitable for solving this type of inverse problem. Therefore, until now, analytical formulas have continued to be important tools for the analysis of coaxial sensors. Furthermore, the application of numerical methods to electromagnetic fields is very challenging because the method strongly affects the operational wavelength and the size of the discrete mesh grid.

Most of the origin publications for those analytical formulations do not describe in detail what are the weaknesses and limitations of their equations. However, among are some analytical formulas which are quite reliable and accurate for low-frequency modeling. In this paper, the detailed results of our investigation of the restrictions and limitations of frequency-domain analytical models for coaxial waveguides are presented, and the reasons for using numerical methods to replace analytical methods are discussed. In addition, the strengths and weaknesses of numerical methods are analyzed and discussed.

\section{Analytical Methods}

2.1. Open-Ended Coaxial Line. The earliest work $[4,5]$ concerned the homogeneous case, in which an air-filled coaxial line with an infinite conducting flange is radiated into the infinite half-space. Simultaneously, the variation expressions for normalized admittance aperture, $\tilde{Y}$, are given by

$$
\begin{aligned}
\tilde{Y} & =\frac{j k_{2}}{k_{1} \ln (b / a)} \int_{0}^{\infty} \frac{d \zeta}{\zeta\left(\zeta^{2}-k_{2}^{2}\right)^{1 / 2}}\left|J_{o}(\zeta a)-J_{o}(\zeta b)\right|^{2} \\
& =\frac{G(0)}{Y_{o}}+j \frac{B(0)}{Y_{o}} .
\end{aligned}
$$

The real part and the imaginary part in (1) are called normalized conductance, $G(0) / Y_{o}$, and susceptance, $B(0) / Y_{o}$, respectively $[4,5]$ :

$$
\begin{aligned}
\frac{G(0)}{Y_{o}}=\frac{k_{2}}{k_{1} \ln (b / a)} \int_{0}^{\pi / 2} \frac{d \theta}{\sin \theta}\left[J_{o}\left(k_{2} a \sin \theta\right)-J_{o}\left(k_{2} b \sin \theta\right)\right]^{2} \\
\frac{B(0)}{Y_{o}}=\frac{k_{2}}{\pi k_{1} \ln (b / a)} \int_{0}^{\pi} d \phi\left\{2 \operatorname{Si}\left(k_{2} a|u|\right)\right. \\
-\operatorname{Si}\left(2 k_{2} a\left|\sin \left(\frac{\phi}{2}\right)\right|\right) \\
-
\end{aligned}
$$

where $u^{2}=a^{2}+b^{2}-2 a b \cos \phi$ and Si are the sine integral or $\operatorname{Si}(x)=\int_{0}^{x} \sin t / t d t$. Presently, a simplified version of (1) introduced in [6] is the most commonly used equation to calculate the admittance of the open-ended coaxial sensor

$$
\tilde{Y}=\frac{j k_{2}^{2}}{\pi k_{1} \ln (b / a)} \int_{a}^{b} \int_{a}^{b} \int_{0}^{\pi} \frac{\exp \left(-j k_{2} r\right)}{r} \cos \phi^{\prime} d \phi^{\prime} d \rho^{\prime} d \rho .
$$

The discontinuity at the open end of the coaxial line is commonly interpreted using admittance expressions (1) and (4) due to the naturally capacitive properties that dominate at the open-end surface $(z=0)$. Finally, analytical equations (1) and (4) can be solved using several classical numerical approaches, such as the series expansion method and the Gaussian quadrature routines. However, the analytical equations only are suitable for ideal conditions in which the component electric field, $E_{z}$, has been neglected in (1) and (4) [4], as shown in Figure 1(a). In actual situations, in addition to components $E_{\rho}$ field, the component $E_{z}$ also is present on the surface $z=0$, as shown in Figure 1(b).

Typically, component $E_{z}$ is neglected to facilitate solving the problem, since components $E_{z}$ involve unknown variables in its formulation [7]. So, to get accurate results from the calculations, modern numerical methods have played an important role in determining the values of unknown variables.

In the early 1980s, the admittance model was simplified by using an equivalent circuit, in which the circuit consists 
of two parallel capacitance terms and one conductance term, $G_{o}[8]$ :

$$
\tilde{Y}_{\text {in }}=\frac{1}{Y_{0}}\left[j \omega\left(C_{1}+\varepsilon_{r} C_{2}\right)+G_{o} \omega^{5 / 2}\right] .
$$

The two capacitances, $C_{1}$ and $C_{2}$ in (5), are the capacitive properties inside the coaxial line near the aperture probe and the capacitive properties in an external sample under test, respectively. However, the two capacitance terms are not sufficient to represent the equivalent circuit for the aperture probe, which interacts with lossy materials at high-frequency operation. In this study, we propose that inductance, $L$, and resistance, $R$, should be taken into account in the circuit model, as shown in Figure 2. In this work, the composition for the $C, R$, and $L$ elements refer to the impedance before it is converted to function admittance. Thus, the normalized input impedance, $\widetilde{Z}_{\text {in }}$, and admittance, $\widetilde{Y}_{\text {in }}$, are given as

$$
\begin{gathered}
\tilde{Z}_{\text {in }}=Y_{o}\left[\frac{1}{j \omega\left(C_{1}+\varepsilon_{r} C_{2}\right)}+j \omega\left(L_{1}+\varepsilon_{r} L_{2}\right)+R\right], \\
\tilde{Y}_{\text {in }}=\frac{1}{\widetilde{Z}_{\text {in }}} .
\end{gathered}
$$

The capacitances $C_{1}$ and $C_{2}$ in (6a) have the same meaning as in (5). The $L_{1}$ and $L_{2}$ are the inductive properties inside the coaxial line near the aperture probe and the inductive properties in an external sample under test, respectively. The $R$ is the resistive properties at the aperture probe. The values of components $C_{1}, L_{1}, L_{2}$, and $R$ are obtained by optimization between the normalized input impedance, $\tilde{Z}_{\text {in }}$, and the finite element simulation results, while the $C_{2}$ values in (5) and (6a) are calculated from $C_{2}=2.38 \varepsilon_{0}(b-a)$ [9].

The comparison of calculated normalized conductance, $G(0) / Y_{o}$, and susceptance, $B(0) / Y_{o}$, using several models and the simulation results (using COMSOL Multiphysics [10], a finite element analysis simulation software) and measurements for water at room temperature are shown in Figures 2 (a) and 2(b), respectively. The calculations for admittance in (4) were conducted by using a $5 \times 5 \times 6$-order Gaussian triple integral method, while (2) and (3) were solved by using the series expansion method with 25 series terms. The dispersive properties of the water in simulation and all admittance calculations were obtained from the Cole-Cole model [11] with $\varepsilon_{s}=78.6, \varepsilon_{\infty}=4.22, \tau=8.8 \mathrm{ps,} \mathrm{and} \alpha=$ 0.013 . The results measured over the frequency range from $0.3 \mathrm{GHz}$ to $18 \mathrm{GHz}$ were obtained by using a Teflon-filled coaxial sensor with $a=0.65 \mathrm{~mm}$ and $b=2.05 \mathrm{~mm}$.

From Figures 2(a) and 2(b), the closed form equation (5) with $C_{1}=0.1 \mathrm{pF}, C_{2}=0.0293 \mathrm{pF}$, and $G_{o}=9 \times 10^{-29}$ is applicable only up to $6 \mathrm{GHz}$, while $(6 \mathrm{a})$ shows that the calculated values are in good agreement with the corresponding simulation results up to $40 \mathrm{GHz}$. The values for the components $C_{1}, L_{1}, L_{2}$, and $R$ in (6a) were given as $C_{1}=$ $0.1 \mathrm{pF}, C_{2}=0.0293 \mathrm{pF}, L_{1}=7 \mathrm{pH}, L_{2}=0.9 \mathrm{pH}$, and $R=$ $2.8 \Omega$. Equations (2), (3), and (4) were solved analytically, but with different approaches. The series expansion solution of (2)-(3) agrees with those corresponding admittance results for frequencies only up to $18 \mathrm{GHz}$ and becomes unstable for higher frequencies. This unstable condition may be due to an inaccuracy of the decimal numeral in the series terms. However, the series expansion solutions can be used efficiently to minimize the run time of the program compared to the Gaussian integral method.

2.2. Monopole Driving from Coaxial Line. For the extendedconductor case, the normalized input impedance, $Z_{\text {in }}$, can be derived using the induced electromagnetic field (EMF) method [12] as

$$
\begin{aligned}
\tilde{Z}_{\text {in }}=\frac{j(0.5) k_{1}}{k_{2} \ln (b / a) \sin ^{2}\left(k_{2} h\right)} \int_{0}^{h} \sin \left[k_{2}(h-z)\right] \\
\times\left[\frac{e^{-j k_{2} R_{1}}}{R_{1}}+\frac{e^{-j k_{2} R_{2}}}{R_{2}}\right. \\
\left.\quad-2 \cos (k h) \frac{e^{-j k_{2} r^{\prime}}}{r^{\prime}}\right] d z .
\end{aligned}
$$

While, in [12], listed out the input impedance, $\tilde{Z}_{\text {in }}$ for monopole driven from coaxial line can be written as

$$
\begin{aligned}
& \tilde{Z}_{\text {in }}= \frac{j(0.5) k_{1}}{k_{2} \ln (b / a) \sin ^{2}\left(k_{2} h\right)} \\
& \times \int_{0}^{h} \sin \left[k_{2}(h-z)\right] \\
& \quad \times\left[\frac{e^{-j k_{2} R_{1}}}{R_{1}}-\cos (k h) \frac{e^{-j k_{2} r^{\prime}}}{r^{\prime}}\right. \\
&\left.\quad-z \sin \left(k_{2} h\right) e^{-j k_{2} r^{\prime}}\left(\frac{j}{r^{\prime 2}}+\frac{1}{k_{2} r^{\prime 3}}\right)\right] d z,
\end{aligned}
$$

where $\varepsilon_{c}$ is the relative permittivity of lossless material filled in coaxial line (at region $z \leq 0$ ). Equations (7) and (8) can be solved easily by Simpson's rules. For extended conductor cases, the energy is released through the extended conductor from the end of the coaxial line, thus the inductive properties are dominant at driving point $(z=0)$. Hence, the analysis and the prediction of the performance of an extended conductor waveguide are often referred as input impedance, $Z_{\text {in }}=R_{\text {in }}+j X_{\text {in }}$, rather than input admittance, $Y_{\text {in }}=G_{\text {in }}+j B_{\text {in }}$.

Figure 3 shows the calculated normalized resistance, $R_{\text {in }} / Z_{o}$, and the normalized reactance, $X_{\text {in }} / Z_{o}$, for air at room temperature using (7) and (8). It was found that (7) and (8) give similar resistance values, $R_{\text {in }} / Z_{o}$, in the frequency range of $300 \mathrm{kHz}$ to $20 \mathrm{GHz}$. Meanwhile, both equations give les consistent values of reactance, and this may be due to the fact that the field distribution near the source point was different between the two models. The impedance integral equations are important in analytical calculations and for numerical solutions. Numerical solutions, such as MoM, cannot be used if the problems have no established analytical integral equation. Moreover, the accuracy of the MoM method is 


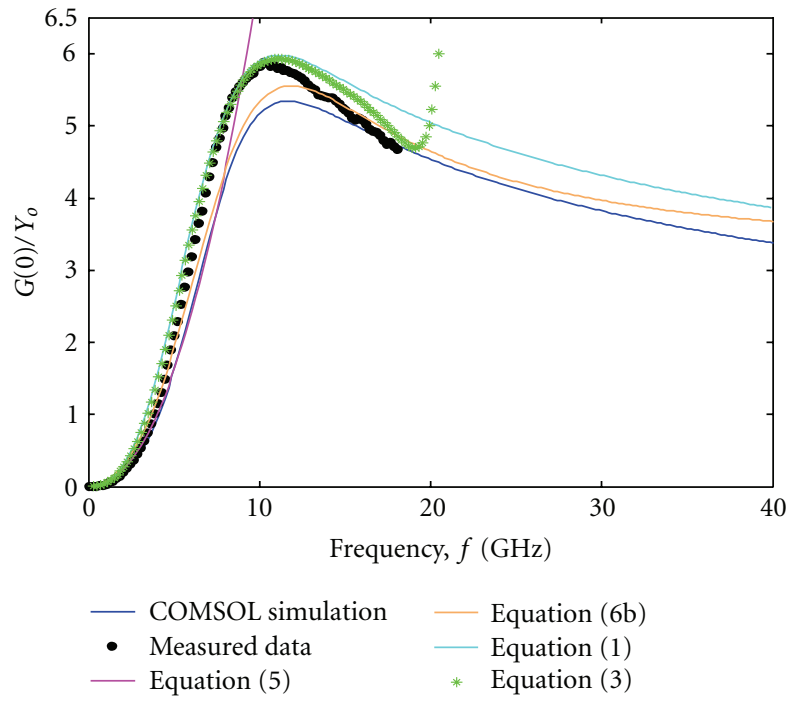

(a)

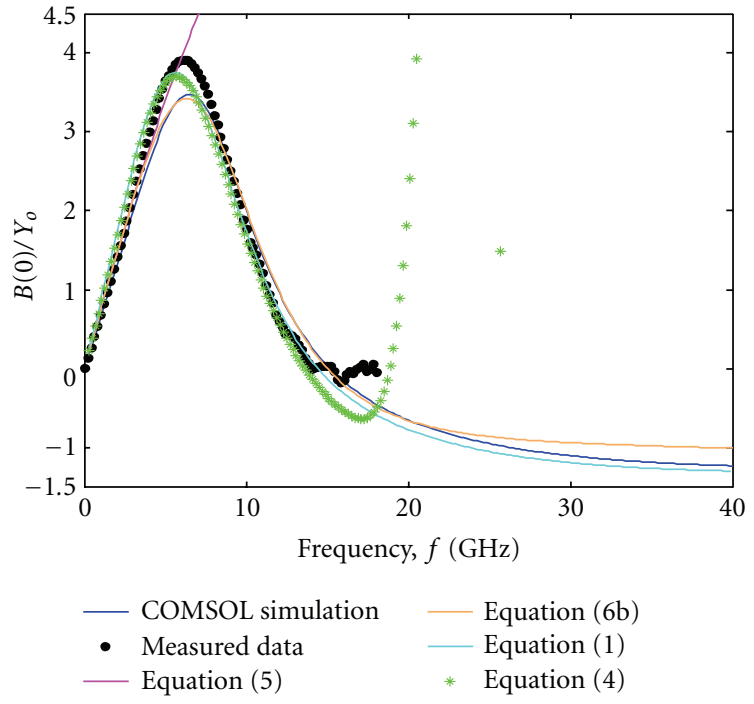

(b)

FIgure 2: Comparison of (a) normalized conductance, $G(0) / Y_{o}$, and (b) normalized susceptance, $B(0) / Y_{o}$, for water at $25^{\circ} \mathrm{C}$ by considering size of probe with $a=0.65 \mathrm{~mm}$ and $b=2.05 \mathrm{~mm}$.

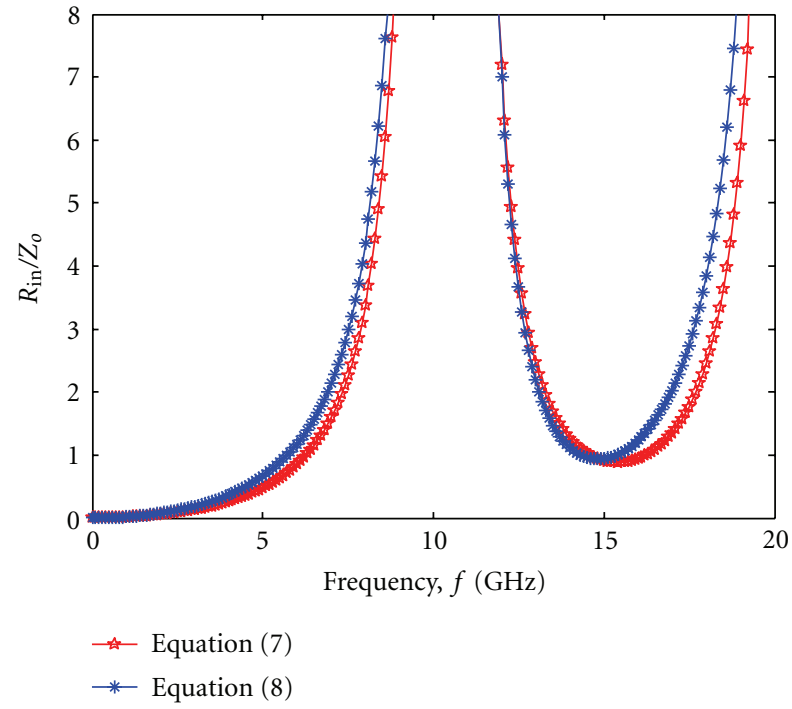

(a)

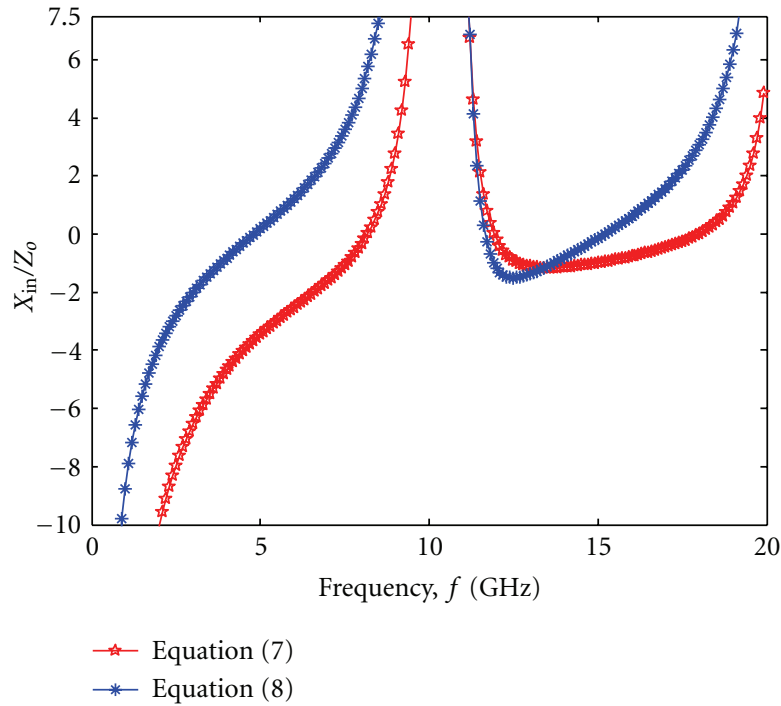

(b)

FIgURE 3: Comparison of calculated normalized resistance, $R_{\mathrm{in}} / Z_{o}$, and normalized reactance, $X_{\mathrm{in}} / Z_{o}$, for air at $25^{\circ} \mathrm{C}$ using $(7)$ and $(8)$ ( $a=0.65 \mathrm{~mm}, b=2.05 \mathrm{~mm}$, and $h=14.32 \mathrm{~mm}$ ).

dependent on the rigor of the analytical integral equation. For instance, (1) and (8) have been used as the governing equations in the MoM method $[7,13,14]$. However, in the MoM solution, the current, $I_{z}$, is an unknown parameter that must be solved by a numerical routine. Instead, in analytical calculations, the current, $I_{z}$, is usually replaced by the sinusoidal current model $[12,13,15]$ in the integral equation before it is solved.
The distribution current, $I_{z}$, substituted in (7) and (8) was assumed to have a convenient sinusoidal form, such as $[12,13,15]$ :

$$
I_{z}=\frac{I(0) \sin k_{2}(h-z)}{\sin \left(k_{2} h\right)},
$$

where $I(0)$ is the amplitude of the driving point current at $z=0$, and it was canceled out in the derivations in (7) and 
(8). Until now, no completely actual distribution current, $I_{z}$, formulation has existed for an arbitrarily sized extended conductor. The current calculated using (9) is clearly the real value, but, in an actual case, the current is a complex value due to the azimuthal current, $I_{\phi}$, contributed by the size of the radius of conductor $a$, as shown in Figure 4(b). This means that (9) is valid only for thin extended conductors. In addition, it cannot be guaranteed that the current distribution will always be sinusoidal in all cases of the extended conductor.

Figure 5 shows the variational of distribution current, $I_{z}$ along the length, $z$, of monopole. The unknown complex current, $I_{z}$, is determined by using point matching MoM with Pocklington's integral equation and frill-generator source $[14,16,17]$. For cylindrical monopole structure, the magnetic field, $\vec{H}$, around the surface monopole which is generated by a current, $I_{z}$, is in an azimuthal, $\phi$, direction, and the relationship is given as $H_{\phi}=I_{z} / 2 \pi a \hat{\phi}$. This means that the imaginary part of current, $I_{z}$, is referring to the current flow in $z$-direction (perpendicular to the $\phi$ direction). While, the real part of $I_{z}$ is represented in the azimuthal current. From Figure 5, it is clear that, at low frequencies $(f<$ $3 \mathrm{GHz}$ ), the value of current, $I_{z}$, is approaching the real number. When the monopole operated at higher frequencies, the distribution current, $I_{z}$, along the length of monopole become complex number.

In addition to the uncertainty of the distribution current, (7) and (8) also have not considered the fringing fields contributed by the electric field, $E_{\rho}$, near the driving point $(z=0)$ and the end of the terminate conductor $(z=h)$. For a short conductor, $(h<10 a)$, driven from a coaxial line, the input impedance is affected significantly by the fringing field at the end of the coaxial line, especially for high-frequency operation [18]. This means that the analytical impedance formulas of (7) and (8) are accurate only for the lowfrequency condition. For the reasons stated, the full wave $\left(H_{\phi}, E_{\rho}\right.$, and $\left.E_{z}\right)$ analysis of the waveguide must be conducted by using a numerical method. In practice, the effects of fringing fields at the driving point are always empirically corrected by capacitance, $C_{f}$, element circuits.

In addition to integration models, the input impedance of the monopole also can be represented by using the lumped-element model [19] and expressed as

$$
\begin{aligned}
Z=\underbrace{j\left(\omega L_{1}-\frac{1}{\omega C_{1}}\right)}_{\text {Low frequencies }}+\underbrace{\frac{1}{j \omega C_{2}+\left(1 /\left(j \omega L_{2}+R_{1}\right)\right)}}_{\text {High frequencies }} \\
+\underbrace{\frac{1}{j \omega C_{3}+\left[1 /\left(j \omega L_{3}+\left(50 j \omega L_{4} /\left(j \omega L_{4}+50\right)\right)\right)\right]}}_{\text {Driving fringing fields }} .
\end{aligned}
$$

From Figures 7(a) and 7(b), the input impedance show that only two peaks of curve line occurred from $300 \mathrm{kHz}$ to $20 \mathrm{GHz}$, the three terms of (10) are sufficient to model the impedance properties cover the frequency range. The first term in (10) describes the characteristics of the impedance at low frequencies, while the second term contributes to the modeling of impedance for high frequencies $(>10 \mathrm{GHz})$. The third term is used to model the equivalent circuit, which is near the driving point for the monopole. This means that if there are three peaks of impedance curve line (See Figure 14) over $300 \mathrm{kHz}$ to $20 \mathrm{GHz}$, up to four terms of lumped-element expression are required. The equivalent circuit and all optimized values of resistance, $R$, capacitance, $C$, and inductance, $L$, elements in (10) for air are shown in Figure 6(b), and the values are accurate up to $20 \mathrm{GHz}$. The $R L C$ values in (10) are obtained by optimizing (10) to the measurement results. At low frequencies, the capacitance term, $C_{1}$, in (10) has played an important role in the modeling, since the input impedance at driving point $(z=0)$ shows the nature of capacitive properties $\left(X_{\mathrm{in}} / Z_{o}\right.$ in negative $(-)$ sign $)$ at a frequency of less than $4 \mathrm{GHz}$, as shown in Figure 7(b).

For instance, a monopole with $h=1.436 \mathrm{~cm}$ driven from the coaxial line, as shown in Figure 6(a), was tested in this work. The comparison of calculated normalized resistance, $R_{\text {in }} / Z_{o}$, and reactance, $X_{\text {in }} / Z_{o}$, using several models, moment method $[14,16,17]$, simulation results (using COMSOL Multiphysics [10] and CST Microwave Studio [20]), and measurements for air at room temperature are shown in Figures 7(a) and 7(b), respectively. Different from the coaxial probe, for a monopole, the fringing field capacitance, $C_{f}$, elements first refer to the admittance before being converted to the impedance function, as follows:

$$
\begin{gathered}
\tilde{Y}=\frac{1}{\text { Equation }(7)}+j \omega C_{f}, \\
\tilde{Z}_{\text {corrected }}=\frac{1}{\tilde{Y}} .
\end{gathered}
$$

In this work, the value of $C_{f}$ was $5.5 \mathrm{pF}$. Figure 7 shows that a $1.436 \mathrm{~cm}$ monopole has been matched with a standard, $50 \Omega$ cable at a frequency of $4.6 \mathrm{GHz}$ and $14.4 \mathrm{GHz}$.

2.3. Coupling Monopole Driving from Coaxial Line. When two different lengths of monopoles are placed closed to each other, as shown in Figure 8(a), the combination of two electromagnetic fields occurs. Now, the input impedance of the monopole is required to consider the self-radiation and mutual-radiation effects.

The mutual normalized impedance, $\widetilde{Z}_{12}$, is well expressed as [21]:

$$
\begin{array}{r}
\tilde{Z}_{12}=\frac{j(0.5) k_{1}}{k_{2} \ln (b / a) \sin \left(k_{2} h_{1}\right) \sin \left(k_{2} h_{2}\right)} \\
\times \int_{0}^{h_{2}} \sin \left[k_{2}\left(h_{2}-z\right)\right] \\
\times\left[\frac{e^{-j k_{2} R_{1}^{\prime}}}{R_{1}^{\prime}}+\frac{e^{-j k_{2} R_{2}^{\prime}}}{R_{2}^{\prime}}\right. \\
\left.-2 \cos \left(k_{2} h_{1}\right) \frac{e^{-j k_{2} R^{\prime}}}{R^{\prime}}\right] d z,
\end{array}
$$




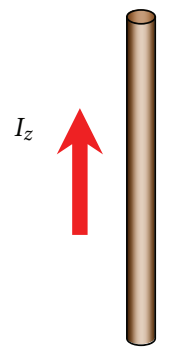

(a)

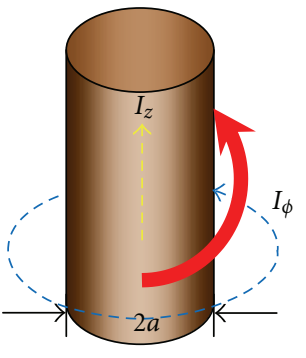

(b)

FIGURE 4: (a) Ideal analytical line form current; (b) Actual situations of current distribution on a cylindrical extended conductor.

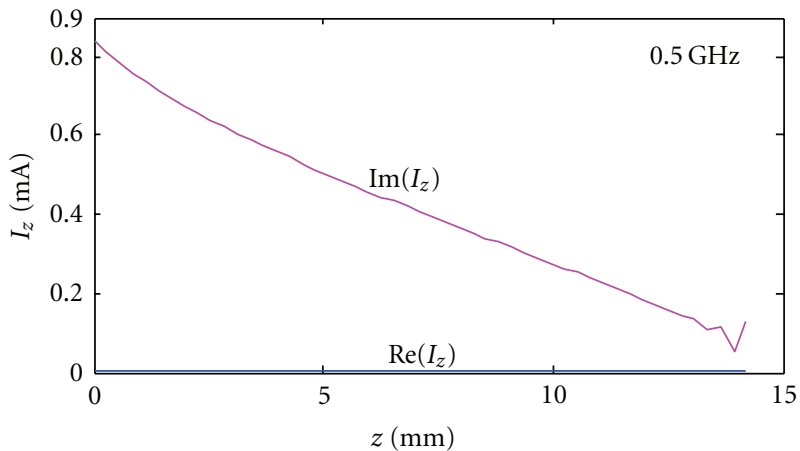

(a)

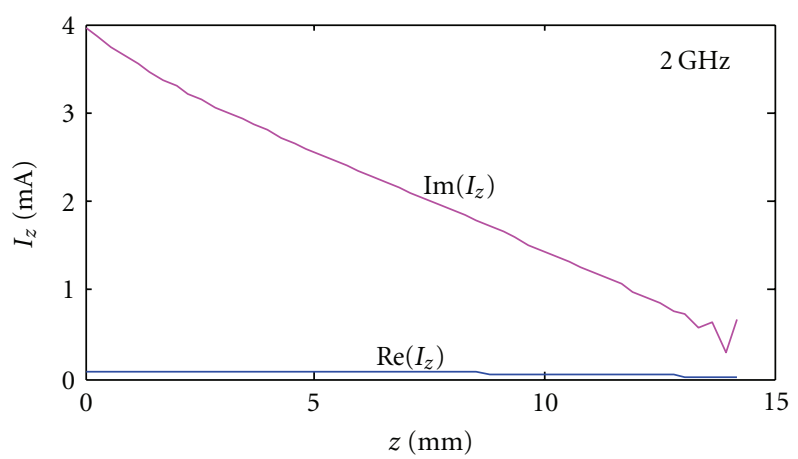

(c)

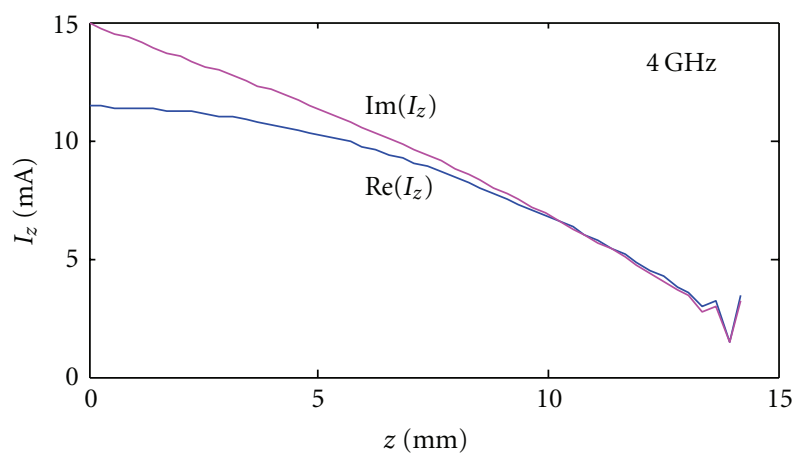

(e)

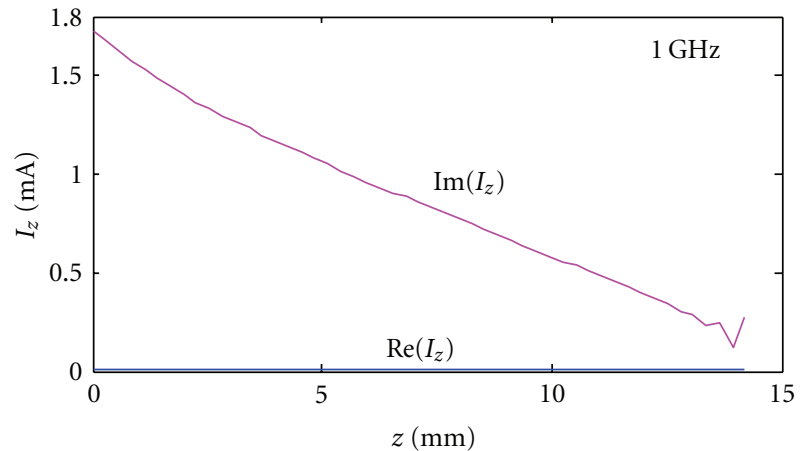

(b)

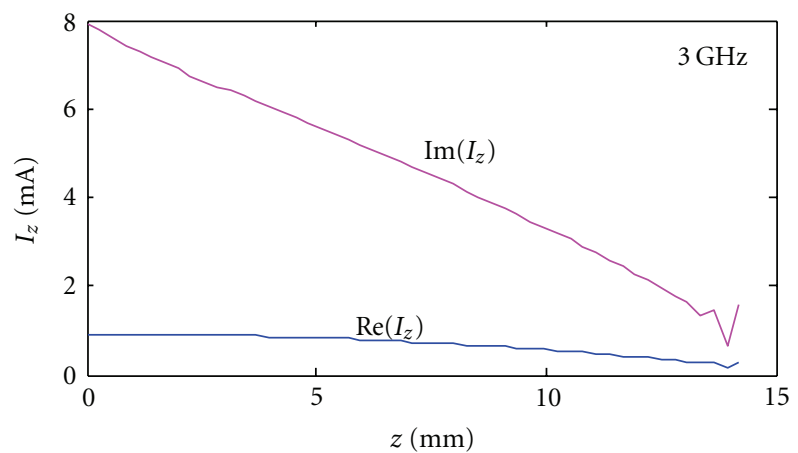

(d)

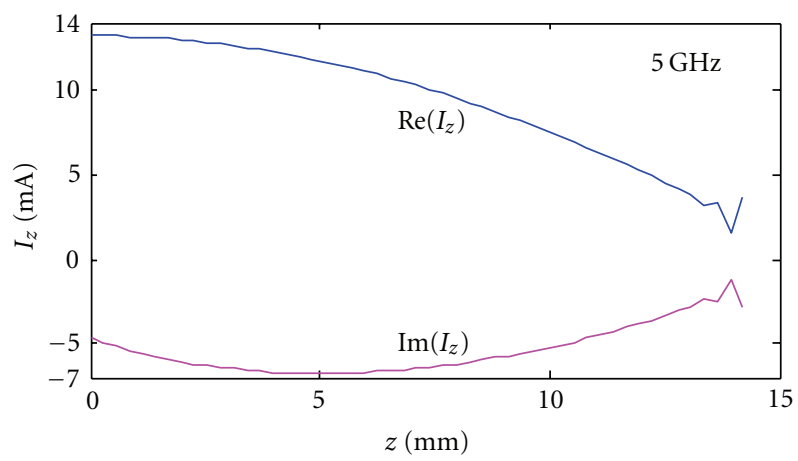

(f)

FIgURE 5: Simulated distribution current, $I_{z}$, on the $1.436 \mathrm{~cm}$ of monopole driving from coaxial line with $a=0.65 \mathrm{~mm}$ and $b=2.05 \mathrm{~mm}$. 


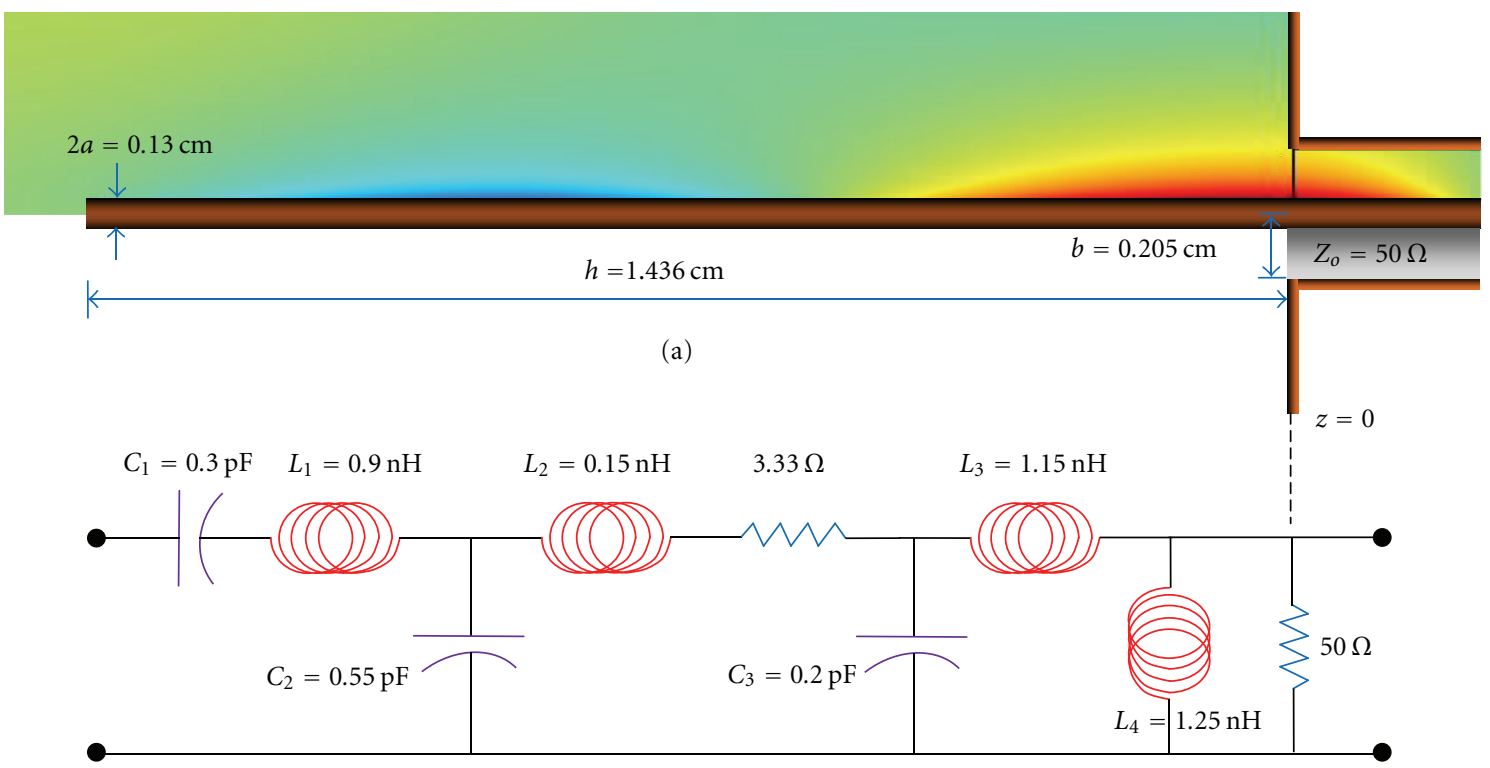

(b)

FigURE 6: Dimensions of monopole and simulated electric fields contour; (b) equivalent circuit for input impedance of monopole at $z=0$.

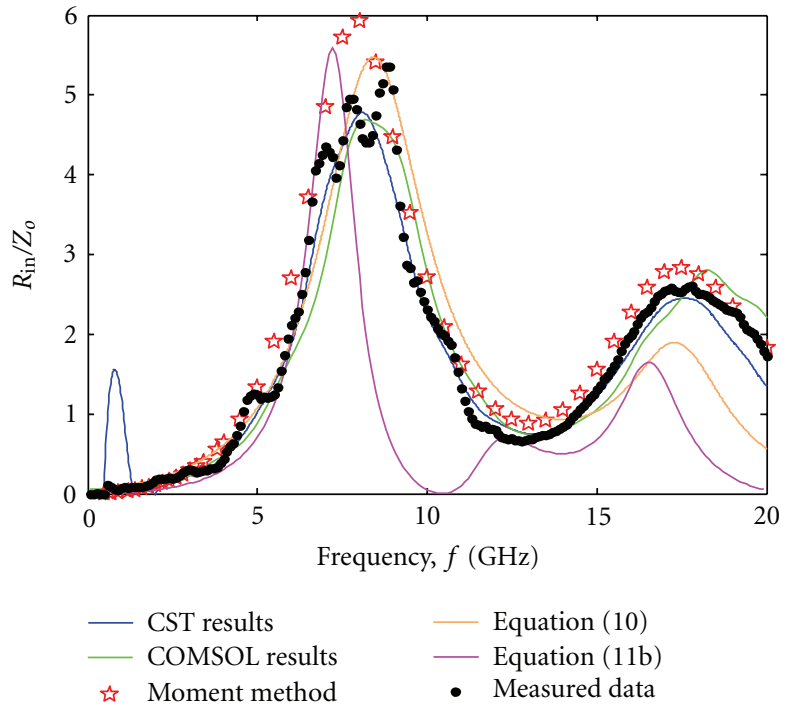

(a)

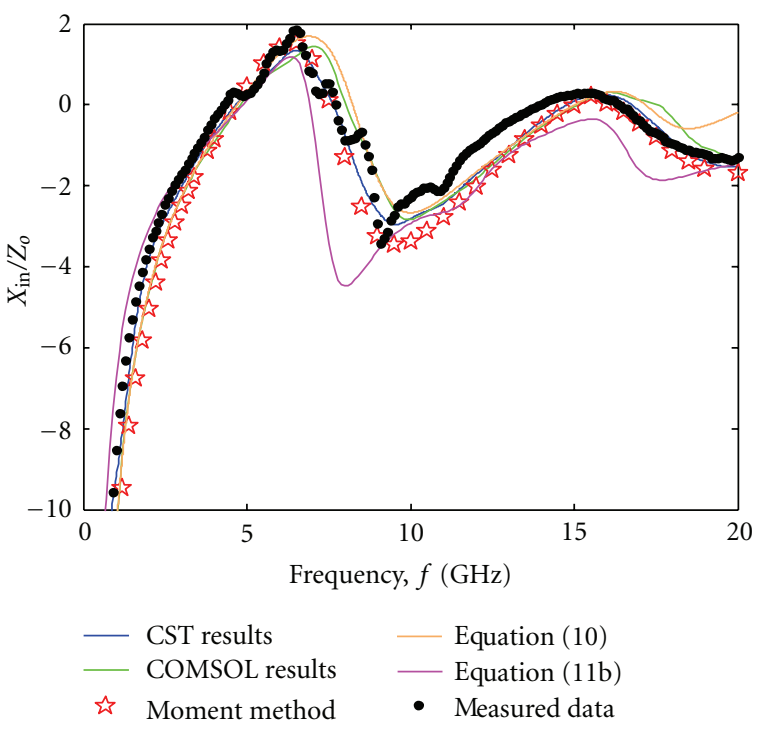

(b)

Figure 7: Comparison of (a) normalized resistance, $R_{\text {in }} / Z_{o}$, and (b) normalized reactance, $X_{\text {in }} / Z_{o}$, for air at $25^{\circ} \mathrm{C}$ by considering size of monopole driving from coaxial line with $a=0.65 \mathrm{~mm}, b=2.05 \mathrm{~mm}$, and $h=14.32 \mathrm{~mm}$.

where $R_{1}^{\prime}=\sqrt{D^{2}+\left(z-h_{1}\right)^{2}}, R_{2}^{\prime}=\sqrt{D^{2}+\left(z+h_{1}\right)^{2}}$, and $R^{\prime}=\sqrt{D^{2}+z^{2}}$. Finally, the input impedance, $\tilde{Z}_{\text {in_port-1 }}$, of monopole 1 can be calculated as $[15,21,22]$ :

$$
\tilde{Z}_{\text {in }- \text { port- } 1}=\tilde{Z}_{11}-\frac{\tilde{Z}_{12}^{2}}{\tilde{Z}_{22}}
$$

where the term $\tilde{Z}_{11}=(7)$ is assumed to be equal to $\tilde{Z}_{22}$, and $\tilde{Z}_{21}$ is equal to $\tilde{Z}_{12}$. Similarly, the input impedance, $Z_{\text {in_port-1 }}$, of the coupling monopole also can be represented by using the lumped-element model and expressed as

$$
\begin{aligned}
Z_{\text {in_port-1 }}= & \underbrace{j\left(\omega L_{1}-\frac{1}{\omega C_{1}}\right)}_{\text {Low frequencies }}+\underbrace{\frac{1}{j \omega C_{2}+\left(1 /\left(j \omega L_{2}+R_{1}\right)\right)}}_{\text {High frequencies }} \\
& +\underbrace{\frac{1}{j \omega C_{3}+\left[1 /\left(j \omega L_{3}+\left(50 j \omega L_{4} /\left(j \omega L_{4}+50\right)\right)\right)\right]}}_{\text {Driving fringing fields }} .
\end{aligned}
$$




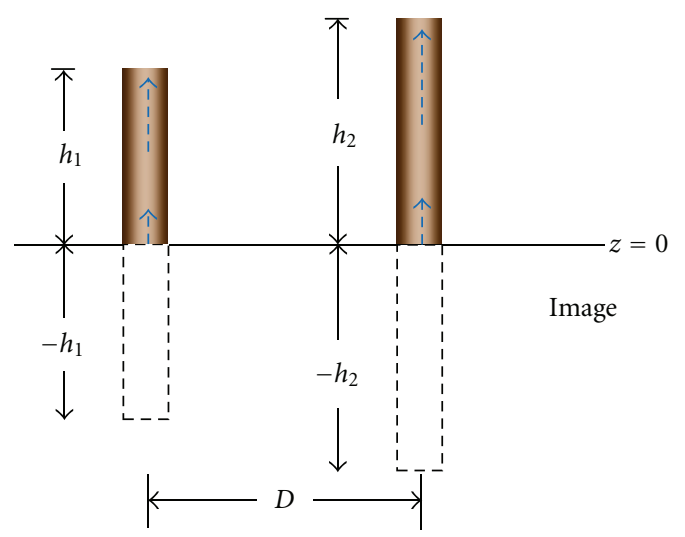

(a)

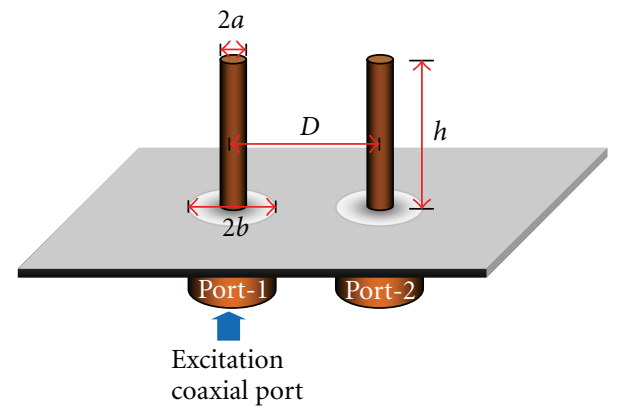

(b)

FIGURE 8: (a) Arbitrary lengths of two parallel, mutually coupled monopoles; (b) actual configuration of coupling monopole with one excitation port.

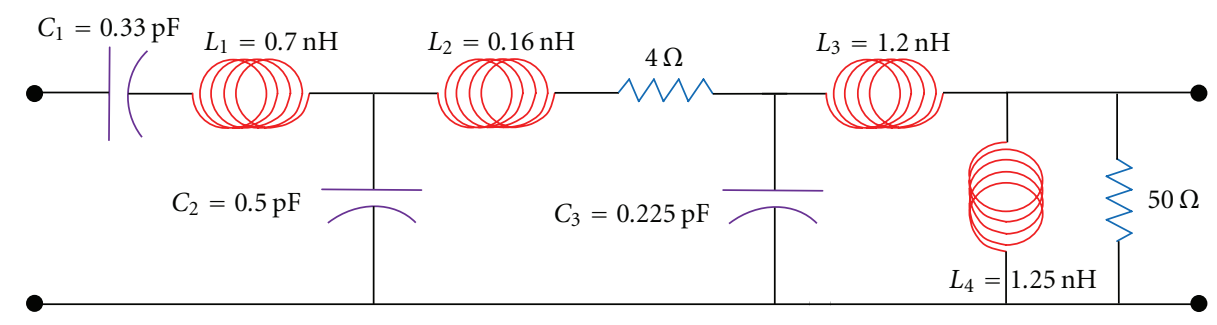

FIGURE 9: Equivalent circuit for input impedance of coupling monopole at port-1 (excitation port).

The equivalent circuit for (14) and its resistance, $R$, capacitance, $C$, and inductance, $L$, elements for air, which are accurate up to $20 \mathrm{GHz}$, are shown in Figure 9. The $R L C$ values in equation (14) are obtained by optimizing (14) to the measurement results. From the comparison of calculated normalized resistance, $R_{\mathrm{in}} / Z_{o}$, and reactance, $X_{\text {in }} / Z_{o}$, using several models, the CST simulation results and measurements for air at room temperature are shown in Figures 10(a) and 10(b), respectively.

The equation for the coupling input impedance, $Z_{\text {in_port-2 }}$, at port-2 can be expressed in terms of input impedance, $Z_{\text {in-port- } 1}$, at excitation port- 1 as

$$
\begin{gathered}
T=\left(1-\frac{\text { Equation }(14)-Z_{o}}{\text { Equation }(14)+Z_{o}}\right) e^{-(\alpha+j \beta) d^{\prime}}, \\
Z_{\text {in_port-2 }}=2 Z_{o}\left(\frac{1-T}{T}\right),
\end{gathered}
$$

where $T$ is the transmission coefficient at parasitic port-2. The exponential term, $e^{-(\alpha+j \beta) d^{\prime}}$, is the transmission factor of the transmitted waves from port- 1 to port- 2 . The symbols $\alpha$ and $\beta$ are the attenuation constant and phase constant, respectively. The equation $d^{\prime}=h_{1}+h_{2}+D+\Delta$ gives the length of the transmission wave from port- 1 to port-2. The normalized input impedances, $Z_{\text {in_port-2 }}=R_{\text {in }}+j X_{\text {in }}$, calculated by using (15b), are plotted and compared with measurement and CST simulation results, as shown in Figure 11.
The normalized resistance, $R_{\mathrm{in}} / Z_{o}$, and reactance, $X_{\mathrm{in}} / Z_{o}$, in Figures 11(a) and 11(b) are calculated with $\alpha=3.18 \times$ $10^{-5} \sqrt{f}$ neper $/ \mathrm{m}, \beta=2 \pi f / c \mathrm{rad} / \mathrm{m}, d^{\prime}=0.044 \mathrm{~m}$ and $Z_{o}=$ $50 \Omega$.

2.4. Coated Conductor Driving from Coaxial Line. A coated monopole is a bare monopole enclosed by a thin cylindrical low-loss dielectric material with an outer radius, $b$, as shown in Figure 12.

In general, the transmission line formulas are adapted easily to the analysis of a coated antenna. The input impedance, $\widetilde{Z}_{\text {in }}$, of the coated monopole at the drive point $(z=0)$ [23-25] is expressed as

$$
\tilde{Z}_{\text {in }}=-j \frac{Z_{c}}{Z_{o}} \cot \left(k_{L} h^{\prime}\right),
$$

where $Z_{o}$ and $Z_{c}$ are the characteristic impedance in the coaxial line and the characteristic impedance for the coated monopole transmission line, respectively, which can be given as:

$$
Z_{c}=\frac{\gamma k_{L}}{2 \pi k_{c}}\left[\ln \left(\frac{b}{a}\right)+\left(\frac{k_{c}}{k_{2}}\right)^{2} \frac{H_{o}^{(2)}\left(k_{2} b\right)}{k_{2} b H_{1}^{(2)}\left(k_{2} b\right)}\right] .
$$

The complex propagation constant, $k_{L}$, is expressed as:

$$
k_{L}=k_{c} k_{2}\left[\frac{H_{o}^{(2)}\left(k_{2} b\right)+k_{2} b \ln (b / a) H_{1}^{(2)}\left(k_{2} b\right)}{k_{c}^{2} H_{o}^{(2)}\left(k_{2} b\right)+k_{2}^{3} b \ln (b / a) H_{1}^{(2)}\left(k_{2} b\right)}\right]^{1 / 2} .
$$




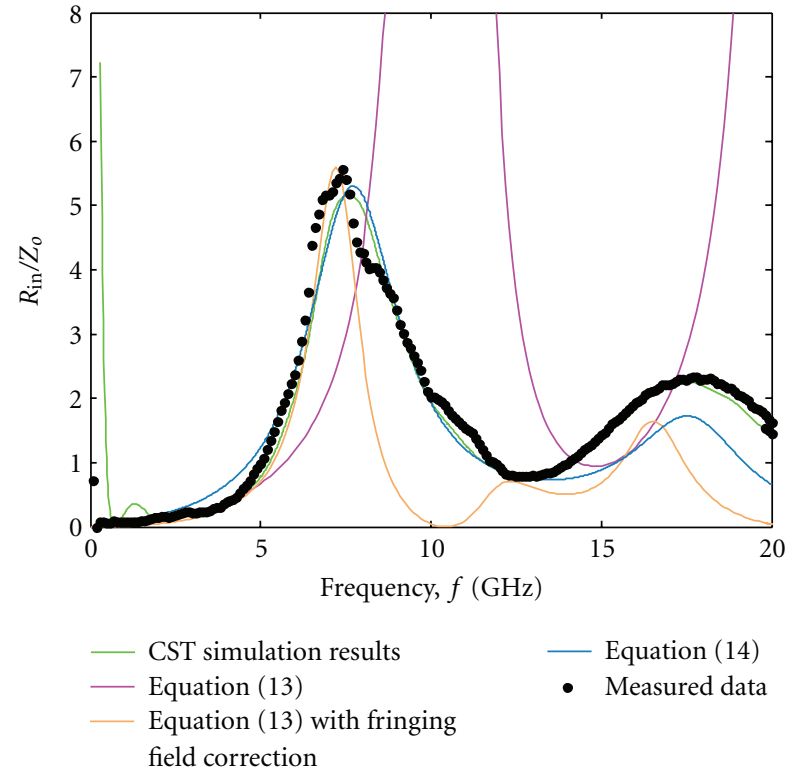

(a)

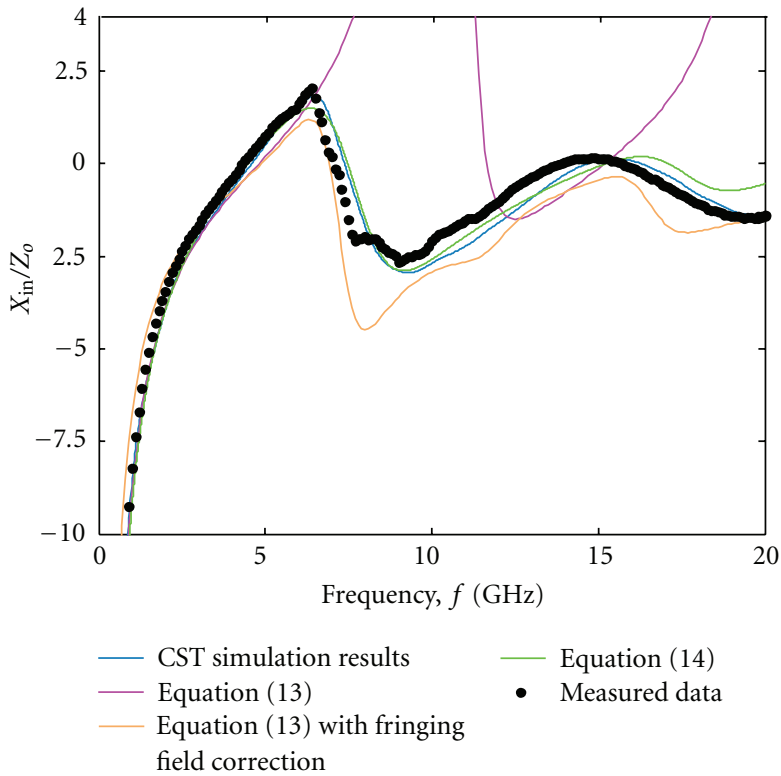

(b)

Figure 10: Comparison of (a) normalized resistance, $R_{\text {in }} / Z_{o}$, and (b) normalized reactance, $X_{\text {in }} / Z_{o}$, of the coupling monopole at excitation port-1 $\left(a=0.65 \mathrm{~mm}, b=2.05 \mathrm{~mm}, h_{1}=h_{2}=14.32 \mathrm{~mm}\right.$, and $\left.D=10 \mathrm{~mm}\right)$.

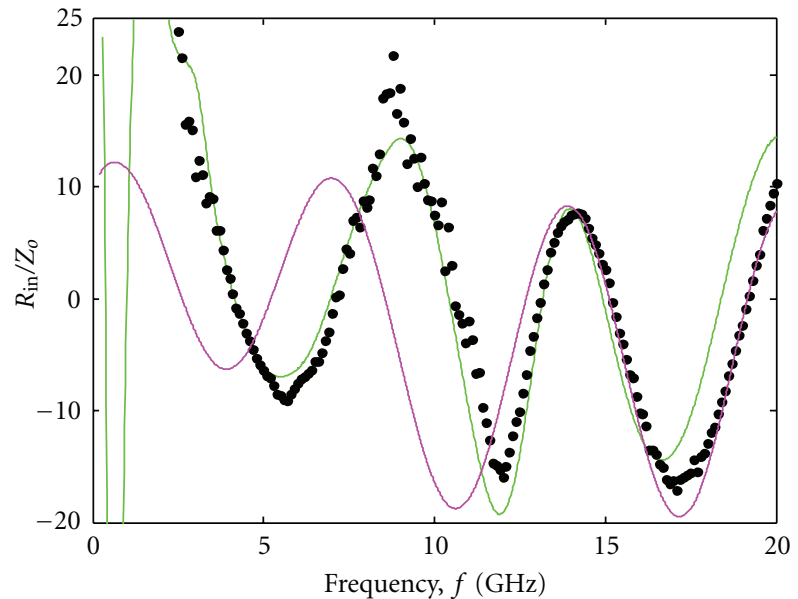

- CST simulation

- Measured data

— Equation (15)

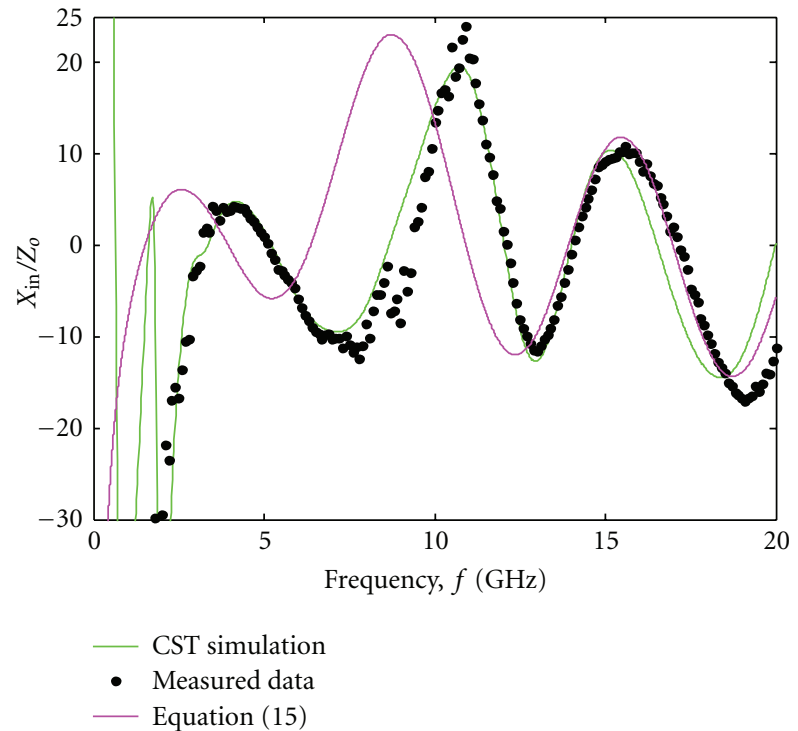

(b)

FIgURE 11: Comparison of (a) normalized resistance, $R_{\text {in }} / Z_{o}$, and (b) normalized reactance, $X_{\text {in }} / Z_{o}$, of the coupling monopole at parasitic port-2 $\left(a=0.65 \mathrm{~mm}, b=2.05 \mathrm{~mm}, h_{1}=h_{2}=14.32 \mathrm{~mm}\right.$, and $\left.D=10 \mathrm{~mm}\right)$.

The transmissionline model requires that the propagation constant, $k_{2}$, of the external medium be much larger than the propagation constant, $k_{c}$, of the insulation [23-25]. Thus, the coated monopole is almost always used in an ambient medium with higher dielectric properties than its insulation, such as soil, seawater, or biological tissue. Moreover, coating material, such as Teflon, functions as a hygienic protector when the monopole is immersed in a biological sample.
However, for the case in Figure 12, the fringing effects at the top end of the monopole are required to consider when its input impedance is calculated using (16). In this study, the effective length, $h^{\prime}$, of the monopole was used to correct the fringing effects, and it is given as

$$
h^{\prime}=h+\frac{\kappa}{\sqrt{f}},
$$




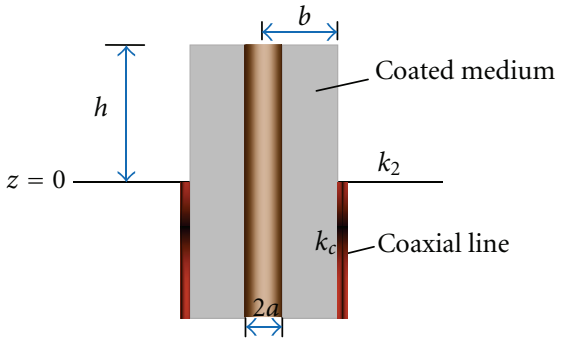

(a)

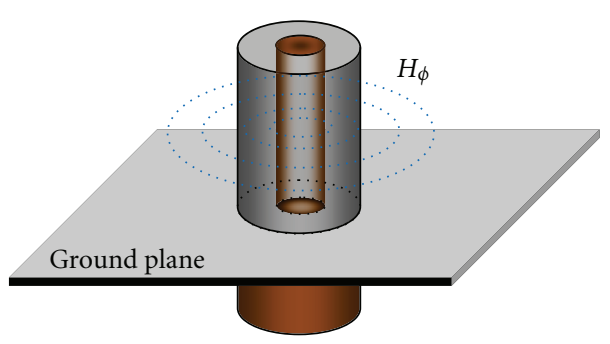

(b)

Figure 12: (a) Two-dimensional and (b) three-dimensional configurations of a coated monopole.

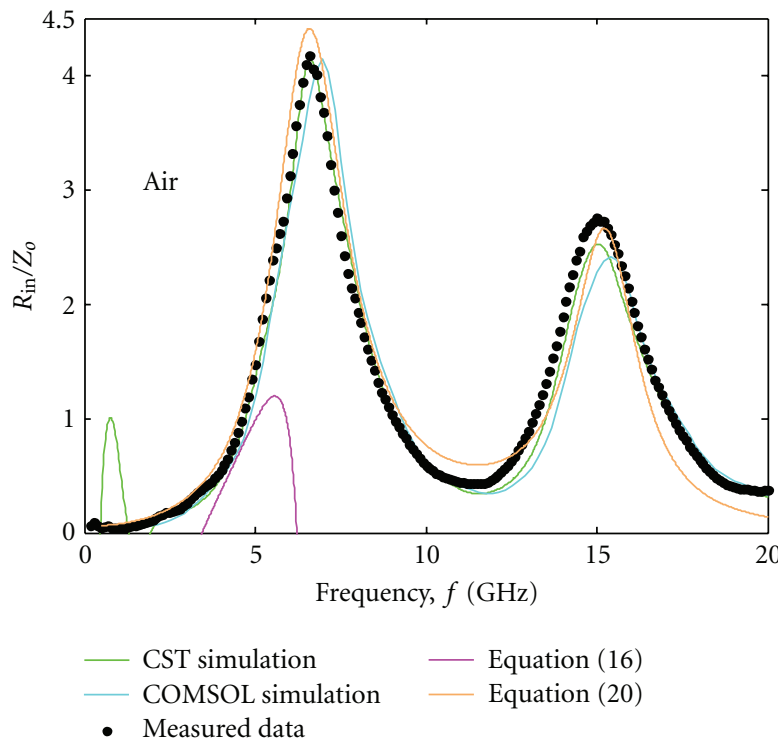

(a)

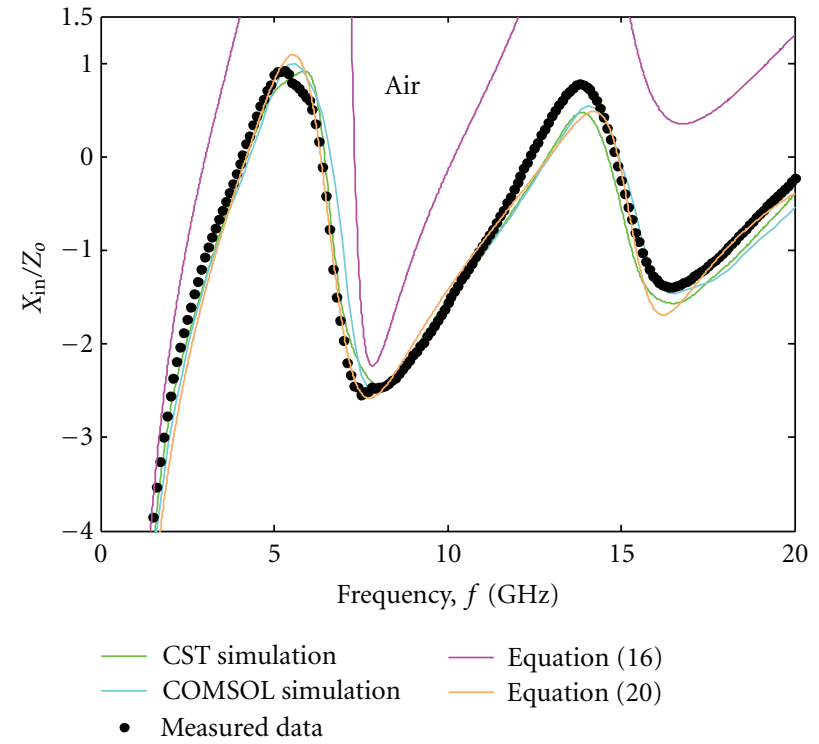

(b)

Figure 13: Comparison of (a) normalized resistance, $R_{\text {in }} / Z_{o}$, and (b) normalized reactance, $X_{\text {in }} / Z_{o}$, for air at $25^{\circ} \mathrm{C}$ by considering size of probe with $a=0.65 \mathrm{~mm}, c=2.05 \mathrm{~mm}$, and $h=13.92 \mathrm{~mm}$.

where $h$ and $f$ are the actual length of the coated monopole and the operational frequency, respectively. The symbol $\kappa$ is a coefficient value which depends on the dimensions of the monopole. The fringing effects are assumed to be inversely proportional to the square root of frequency. Similarly, the equivalent circuit can be used to represent the input impedance properties of the coated monopole, as shown in Figure 12, and the corresponding formulations are expressed as (20):

$$
\begin{aligned}
Z_{\text {in }}= & j\left(\omega L_{T}-\frac{1}{\omega C_{T}}\right)+\sum_{n=1}^{m}\left[\frac{1}{j \omega C_{n}+\left(1 /\left(j \omega L_{n}+R_{n}\right)\right)}\right] \\
& +\frac{1}{j \omega C_{B}+\left[1 /\left(j \omega L_{B}+\left(50 j \omega L_{B}^{\prime} /\left(j \omega L_{B}^{\prime}+50\right)\right)\right)\right]} .
\end{aligned}
$$

Figures 13 and 14 show the calculated normalized resistance, $R_{\text {in }} / Z_{o}$, and reactance, $X_{\text {in }} / Z_{o}$, respectively, using several
TABLE 1: RCL component values in (20) for air and water samples.

\begin{tabular}{lcc}
\hline$R C L$ components & Air & Water \\
\hline$C_{B}$ & $0.33 \mathrm{pF}$ & $0.70 \mathrm{pF}$ \\
$C_{T}$ & $0.40 \mathrm{pF}$ & $2.50 \mathrm{pF}$ \\
$C_{1}$ & $0.54 \mathrm{pF}$ & $0.65 \mathrm{pF}$ \\
$C_{2}$ & - & $0.45 \mathrm{pF}$ \\
$L_{B}$ & $1 \mathrm{nH}$ & $1 \mathrm{nH}$ \\
$L_{B}^{\prime}$ & $1.1 \mathrm{nH}$ & $2.4 \mathrm{nH}$ \\
$L_{T}$ & $0.5 \mathrm{nH}$ & $0.35 \mathrm{nH}$ \\
$L_{1}$ & $0.2 \mathrm{nH}$ & $0.32 \mathrm{nH}$ \\
$L_{2}$ & - & $0.16 \mathrm{nH}$ \\
$R_{1}$ & $2.9 \Omega$ & $5.56 \Omega$ \\
$R_{2}$ & - & $4.0 \Omega$ \\
\hline
\end{tabular}

models and the COMSOL simulation results and measurements for air and water at room temperature $\left(25 \pm 1^{\circ} \mathrm{C}\right)$ over 


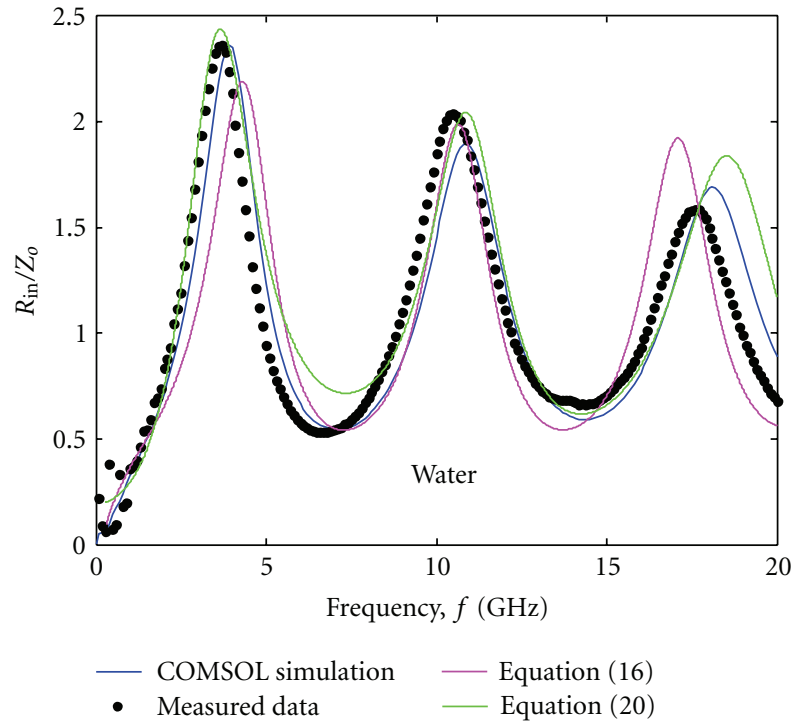

(a)

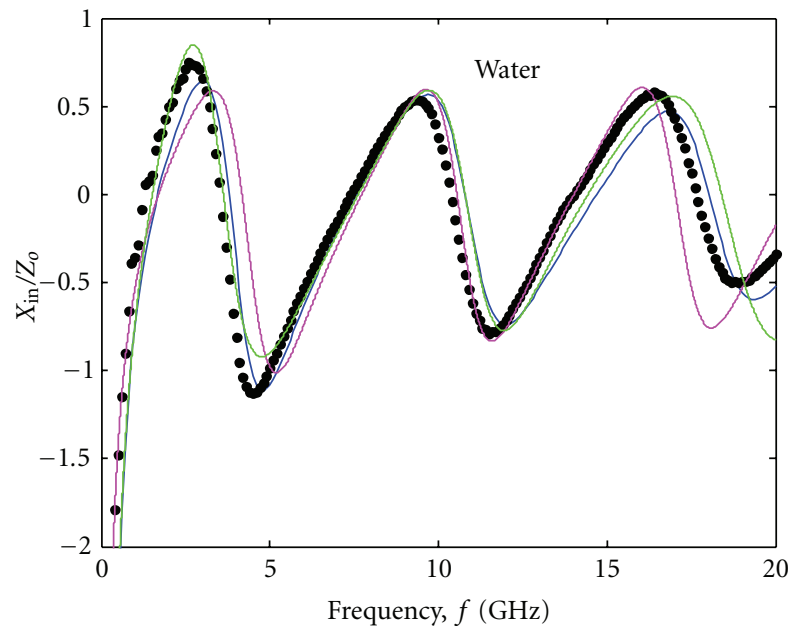

$\begin{array}{ll}\text { - COMSOL simulation } & \text { Equation (16) } \\ \text { Measured data } & \text { Equation (20) }\end{array}$

(b)

Figure 14: Comparison of (a) normalized resistance, $R_{\text {in }} / Z_{o}$, and (b) normalized reactance, $X_{\text {in }} / Z_{o}$, for water at $25^{\circ} \mathrm{C}$ by considering size of probe with $a=0.65 \mathrm{~mm}, c=2.05 \mathrm{~mm}$, and $h=13.92 \mathrm{~mm}$.

the frequency range from $300 \mathrm{kHz}$ to $20 \mathrm{GHz}$. The results were measured using a Teflon-coated monopole driven from coaxial line with $a=0.65 \mathrm{~mm}, c=2.05 \mathrm{~mm}$, and $h=$ $13.92 \mathrm{~mm}$. The values for component resistor, $R$, reactor, $L$, and capacitor, $C$, in (20) for air and water are listed in Table 1, which provides the accurately calculated impedance up to $20 \mathrm{GHz}$. The $R L C$ values in (20) are obtained by optimizing (20) to the measurement results. As expected, compared to the water sample, the calculated normalized resistance, $R_{\text {in }} / Z_{o}$, and reactance, $X_{\text {in }} / Z_{o}$, for air using (16) do not agree well with the measurements and simulation results. The propagation constant, $\gamma$, at the boundary between the coated medium and the external medium is $\gamma= \pm k_{o} \sqrt{\varepsilon_{r}-\varepsilon_{c}}$, thus the tendency properties for the air case $\left(k_{2}<k_{c}\right)$ are different those for the water case $\left(k_{2}>k_{c}\right)$. When $k_{2}<k_{c}$, the waves decay in the normal direction to the interface between the coated dielectric and external medium due to the fact that the corresponding propagation constant, $\gamma$, is imaginary.

\section{Conclusions}

In this work, the coaxial waveguide was used as an example of the problem of linking electromagnetic theory with practical modeling, since the coaxial slot waveguides have been used as antennas over the past of 70 years. Recently, many scientific applications have involved this kind of waveguide. For instance, an open-ended coaxial probe was applied as a dielectric probe to measure the dielectric properties of the material being tested. In addition, the dielectric-coated monopole was used for hyperthermia treatment, and the coupler monopole was designed to be an array antenna. Hence, many models have been developed for the coaxial waveguide, and those models have been modified for use in modeling other devices. In particular, the sinusoidal current model is also used for planar waveguides. In this study, the accuracy of frequency-domain analytical models was tested by acquiring measurements and numerical simulation results. We found that the semiempirical equivalent circuit modeling worked successfully, covered a wide frequency range, and was very useful in the design of circuits that matched the waveguides. Although the analytical models are less accurate compare to numerical method, it provides significant rapid and economize computation. Implicitly, the analytical models still retain the academy valuable, especially for who has preliminary study of the antenna modeling.

\section{Acknowledgment}

This study was supported by the Fundamental Research Grant Scheme (FRGS) Phase 2/2009 from Ministry of Higher Education Malaysia under Project nD. 78486.

\section{References}

[1] K. S. Yee, "Numerical solution of initial boundary value problems involving Maxwell's equations in isotropic media," IEEE Transactions on Antennas and Propagation, vol. 14, pp. 302307, 1966.

[2] R. F. Harrington, "Matrix methods for field problems," Proceedings of the IEEE, vol. 55, pp. 136-149, 1967.

[3] P. P. Silvester, "Finite element solution of homogeneous waveguide problems," Alta Frequenza, vol. 38, pp. 313-317, 1969.

[4] H. Levine and C. H. Papas, "Theory of the circular diffraction antenna," Journal of Applied Physics, vol. 22, no. 1, pp. 29-43, 1951.

[5] N. Marcuvitz, Waveguide Handbook, Boston Technical Publishers, Boston, Mass, USA, 1964.

[6] D. K. Misra, "A quasi-static analysis of open-ended coaxial lines," IEEE Transactions on Microwave Theory and Techniques, vol. 35, no. 10, pp. 925-928, 1988. 
[7] R. D. Nevels, C. M. Butler, and W. Yablon, "The annular slot antenna in a lossy biological medium," IEEE Transactions on Microwave Theory and Techniques, vol. 33, no. 4, pp. 314-319, 1985.

[8] M. A. Stuchly, M. M. Brady, S. S. Stuchly, and G. Gajda, "Equivalent circuit of an open-ended coaxial line in a lossy dielectric," IEEE Transactions on Instrumentation and Measurement, vol. 31, no. 2, pp. 116-119, 1982.

[9] G. B. Gajda and S. S. Stuchly, "Numerical analysis of openended coaxial lines," IEEE Transactions on Microwave Theory and Techniques, vol. 31, no. 5, pp. 380-384, 1983.

[10] COMSOL Multiphysics, "RF module model library version 3.5a," COMSOL AB, TegnŽrgatan, Sweden, 2008.

[11] A. Nyshadham, C. L. Sibbald, and S. S. Stuchly, "Permittivity measurements using open-ended sensors and reference liquid calibration - an uncertainty analysis," IEEE Transactions on Microwave Theory and Techniques, vol. 40, no. 2, pp. 305-314, 1992.

[12] M. M. Weiner, S. P. Cruze, C. C. Li, and W. J. Wilson, Monopole Elements on Circular Ground Plane, Artech House, New York, NY, USA, 1987.

[13] D. K. Misra, "A study on coaxial line excited monopole probes for In-Situ permittivity measurements," IEEE Transactions on Instrumentation and Measurement, vol. 30, pp. 46-51, 1987.

[14] C. A. Balanis, Advanced Engineering Electromagnetic, John Wiley \& Sons, New York, NY, USA, 1989.

[15] R. W. P. King, R. B. Mack, and S. Sandler, Arrays of Cylindrical Dipoles, Cambridge University Press, Cambridge, UK, 1968.

[16] W. C. Gibson, The Method of Moments in Electromagnetics, Chapman \& Hall/CRC, London, UK, 2008.

[17] R. Garg, Analytical and Computational Methods in Electromagnetics, Artech House, Norwood, Mass, USA, 2008.

[18] K. Y. You, Z. Abbas, K. Khalid, and N. F. Kong, "Improved formulation for admittance of thin and short monopole driving from coaxial line into dissipative media," IEEE Antennas and Wireless Propagation Letters, vol. 8, pp. 1246-1249, 2009.

[19] B. S. Yarman, Design of Ultra Wideband Antenna Matching Networks, Springer, Berlin, Germany, 2008.

[20] CST Microwave Studio, "CST Microwave Studio-Workflow \& Solver Overview," CST Computer Simulation Technology, Darmstadt, Germany, 2008.

[21] R. E. Collin, Antennas and Radiowave Propagation, McGrawHill, New York, NY, USA, 1985.

[22] E. E. Altshuler, "Self- and mutual impedances of travelingwave linear antennas," IEEE Transactions on Antennas and Propagation, vol. 37, no. 10, pp. 1312-1316, 1989.

[23] L. Kuan Min, "Chapter 11: insulated linear antenn," in Research Topics in Electromagnetic Wave Theory, J. A. Kong, Ed., John Wiley \& Sons, New York, NY, USA, 1967.

[24] R. W. P. King, S. R. Mishra, K. M. Lee, and G. S. Smith, "The insulated monopole: admittance and junction effects," IEEE Transactions on Antennas and Propagation, vol. 23, no. 2, pp. 172-177, 1975.

[25] T. W. Hertel and G. S. Smith, "The insulated linear antennarevisited," IEEE Transactions on Antennas and Propagation, vol. 48, no. 6, pp. 914-920, 2000. 

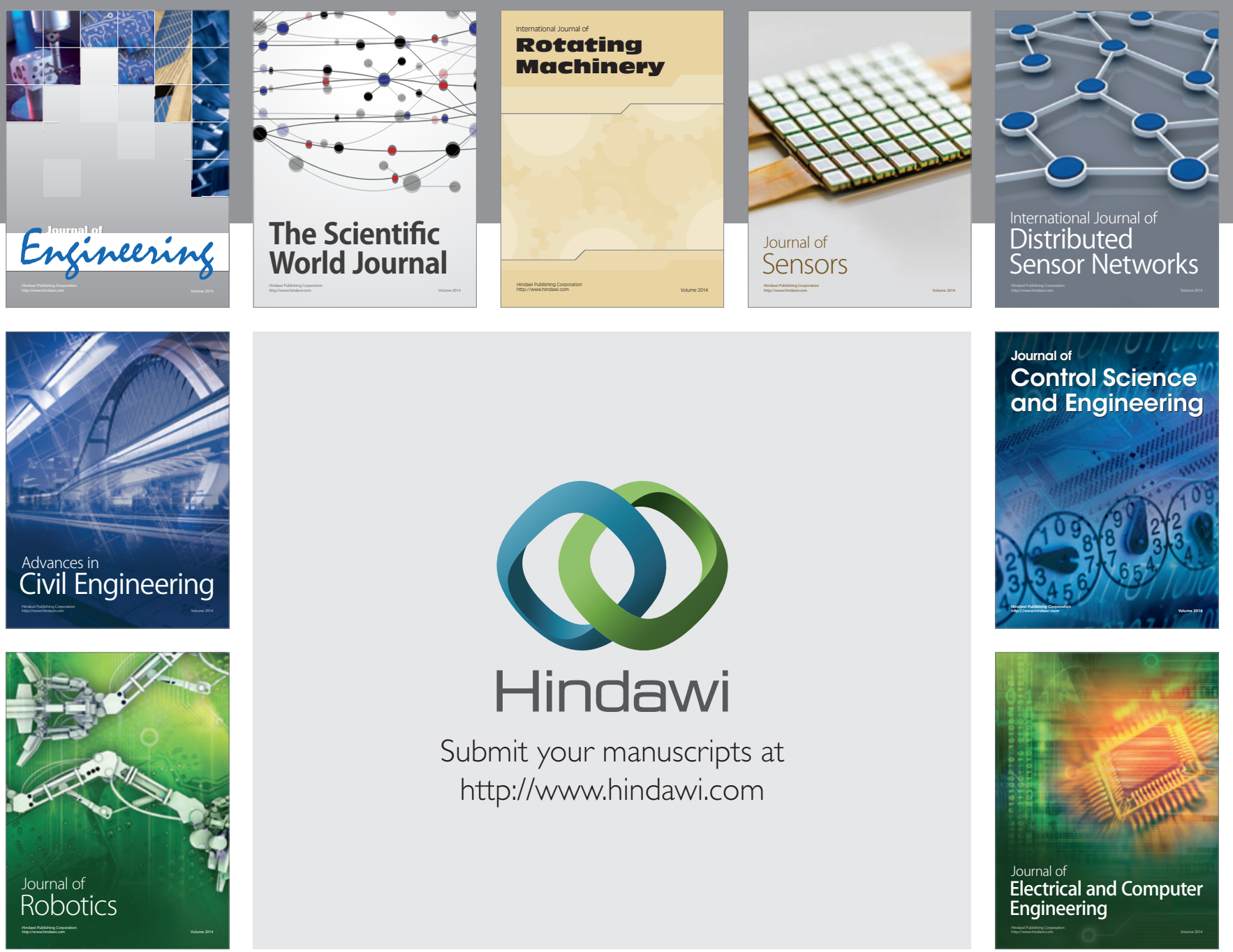

Submit your manuscripts at

http://www.hindawi.com
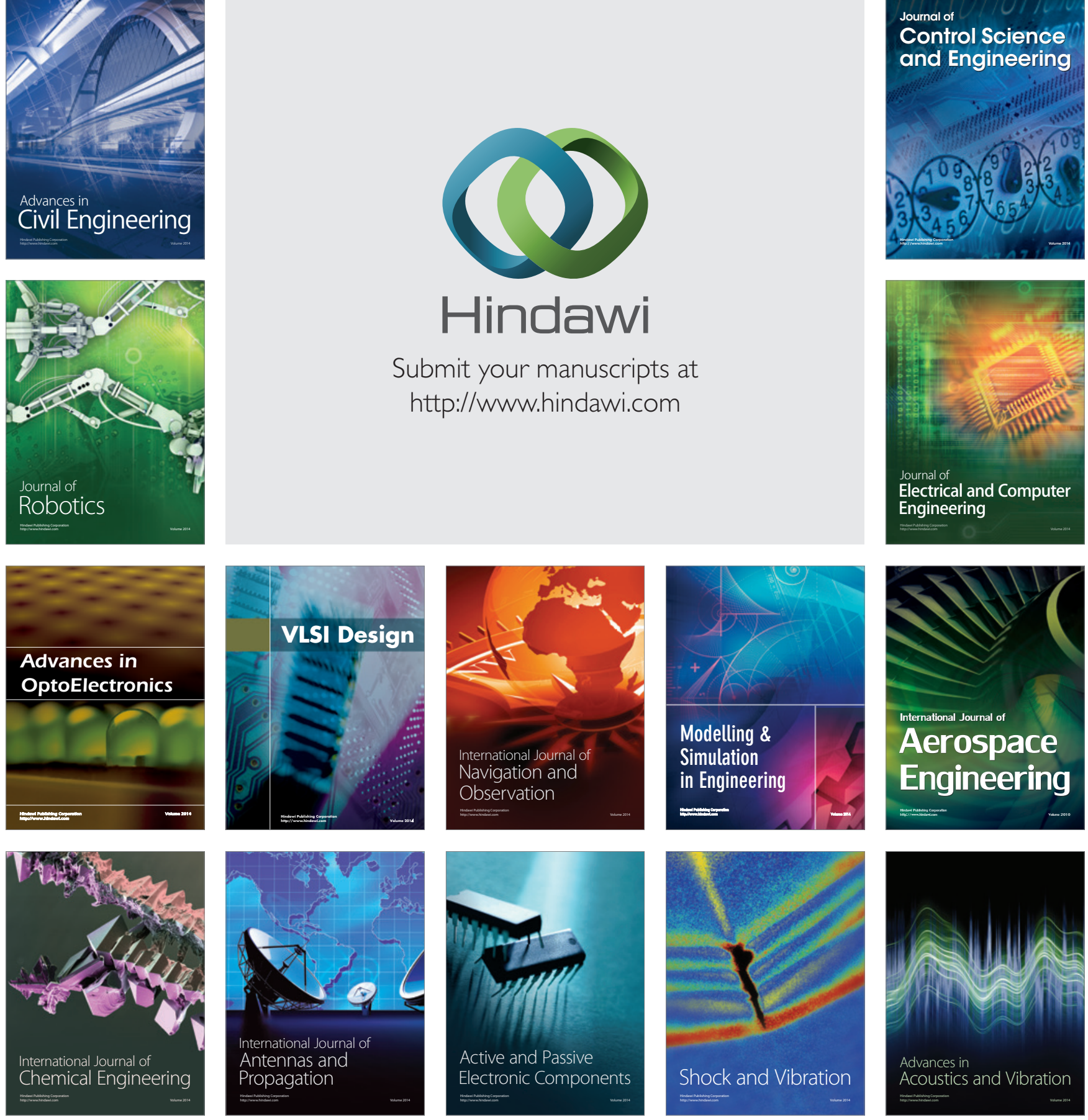